\title{
A New and Fast Real-time Implementation of 2-D DCT
}

\author{
Jianmin Jiang \\ School of Engineering, Bolton Institute, United Kingdom
}

\begin{abstract}
A new and fast $4 \times 4 \mathrm{DCT}$ algorithm is proposed in this paper. This algorithm classifies the input 2-D pixel data into four groups, each of which is then further rearranged into 1-D DCT transform. Therefore, the computation of 2-D DCT can be implemented by four 1-D DCTs. As a result, the efficiency of 2-D DCT algorithm is dependent on the 1-D DCT algorithm adopted, and all the existing fast algorithms for 1-D DCT can be directly applied to further optimise the algorithm design. The proposed algorithm can also be extended to compute general 2-D DCT by a recursive procedure. Specific algorithm flowchart and design is also included and described.
\end{abstract}

\section{Introduction}

Ever since the discrete cosine transform (DCT) was discovered in 1970's, it has been widely accepted as one of the major means for image signal processing and filtering. Its application is particularly widespread on image coding in communication technology. In the image compression standards developed by JPEG and MPEG, DCT is used as one of the most important stages to process the input pixel data.

Research on DCT of $4 \times 4,8 \times 8,16 \times 16$ blocks has been very fruitful [1-3]. All the developments can be summarized into two categories. One is to address the two dimensional DCT directly by investigating the algebraic structure of DCT's and reducing the arithmetic complexity of its computations [4]. The second category features the development of various fast algorithms for one dimensional DCT. Thus, the two dimensional DCT can be addressed by considering its rows and columns individually. The work covered in this paper, however, put forward a novel idea in designing the fast 2-D DCT algorithm by one dimensional approach.

\section{1-D Transformation of 2-D DCT}

In this section, a one dimensional approach is developed to tackle the computation of $4 \times 4$ DCT's. This development can also be used to compute $N \times N$ DCT recursively. The $2^{\mathrm{m}} \times 2^{\mathrm{m}} \mathrm{DCT}$, for example, can be computed by four $2^{\mathrm{m}-1} \times 2^{\mathrm{m}-1} \mathrm{DCT}$. This procedure goes on until we get to the basic $4 \times 4 \mathrm{DCT}$.

Let $[\mathrm{g}]$ be an $4 \times 4$ matrix representing the two-dimensional input pixel data and $[\mathrm{G}]$ be the 2-D DCT of $[\mathrm{g}]$. Then the uv-element of $[\mathrm{G}]$ is given by[1]: 


$$
G(u, v)=\frac{2 c(u) c(v)}{\sqrt{(4 \times 4)}} \sum_{m=0 m=0}^{3} \sum_{m x} \cos \left\{\frac{(2 m+1) u \pi}{8}\right\} \times \cos \left\{\frac{(2 n+1) v \pi}{8}\right\}
$$

where:

$$
c(k)=\left\{\begin{array}{cc}
\frac{1}{\sqrt{2}} & k=0 \\
1 & \text { otherwise }
\end{array}\right.
$$

The above equation can be further rearranged into:

$$
G(u, v)=\frac{c(u) c(v)}{4} \sum_{0}^{3} \sum_{0}^{3} g_{m x}\left\{\cos \frac{(2 m+1) u \pi+(2 n+1) v \pi}{8}+\cos \frac{(2 m+1) u \pi-(2 n+1) v \pi}{8}\right\}
$$

If one variable in the two dimensional transform over $m$ and $n$ can be represented by another variable, the two dimensional DCT can be changed into 1-D DCT. From equation (2), it can be seen that if $(2 n+1)$ can be replaced by $p(2 m+1), p$ is any integer, the transform becomes two 1-D DCT over $m$ for $(u+p v)$ and (u-pv). To obtain a suitable relationship between $m$ and $n$, let $n=m+x$, hence:

$$
2 n+1=2 m+2 x+1=2 m+1+2 x
$$

As $(2 n+1)$ should be replaced by $p(2 m+1)$, $x$ can be determined by:

$$
x=\frac{p(2 m+1)-(2 m+1)}{2}=\frac{(p-1)(2 m+1)}{2}
$$

If $\mathrm{p}$ is further replaced by $2 \mathrm{k}+1, \mathrm{x}$ is of the form:

$$
x=k(2 m+1)
$$

where $\mathrm{k}=0, \pm 1, \pm 2, \ldots$

Thus, $\mathrm{n}$ is represented by:

$$
n=m+x=m+k(2 m+1)=(2 k+1) m+k=p m+k
$$


As $m$ varies from 0 to 3 in $4 \times 4$ DCT, the accurate expression of $n$ should be:

$$
n=p m+k \quad(\operatorname{modulo} 4)
$$

The relationship between $\mathrm{n}$ and $\mathrm{m}$ can be summarized in Table I. From Table I, it can be observed that among all the groups, only four groups are independent. In other words, no matter how the values of $\mathrm{k}$ and $\mathrm{p}$ varies, all the groups obtained can be described by only four groups: $x_{0}, x_{1}, x_{2}$ and $x_{3}$.

Table I 1-D Transform of Input Pixel

\begin{tabular}{|c|c|c|c|c|}
\hline$k$ & $p$ & $n$ & $n(\bmod 4)$ & $x_{k}$ \\
\hline 0 & 1 & $\mathrm{~m}$ & $\mathrm{~m}$ & $x_{0}=\left\{g_{00}, g_{11}, g_{22}, g_{33}\right\}$ \\
\hline 1 & 3 & $3 m+1$ & $3 m+1(\bmod 4)$ & $x_{1}=\left\{g_{01}, g_{10}, g_{23}, g_{32}\right\}$ \\
\hline 2 & 5 & $5 m+2$ & $\mathrm{~m}+2(\bmod 4)$ & $x_{2}=\left\{g_{02}, g_{13}, g_{20}, g_{31}\right\}$ \\
\hline 3 & 7 & $7 m+3$ & $3 m+3(\bmod 4)$ & $x_{3}=\left\{g_{03}, g_{12}, g_{21}, g_{30}\right\}$ \\
\hline 4 & 9 & $9 m+4$ & $\mathrm{~m}$ & $x_{0}=\left\{g_{00}, g_{11}, g_{22}, g_{33}\right\}$ \\
\hline 5 & 11 & $11 \mathrm{~m}+5$ & $3 m+1(\bmod 4)$ & $x_{1}=\left\{g_{01}, g_{10}, g_{23}, g_{32}\right\}$ \\
\hline$\ldots$ & $\ldots$ & $\ldots$ & $\ldots$ & $\ldots$ \\
\hline-1 & -1 & $-m-1$ & $-m-1$ & $x_{3}=\left\{g_{03}, g_{12}, g_{21}, g_{30}\right\}$ \\
\hline-2 & -3 & $-3 m-2$ & $-3 m-2(\bmod 4)$ & $x_{2}=\left\{g_{02}, g_{13}, g_{20}, g_{31}\right\}$ \\
\hline$\ldots$ & $\ldots$ & $\ldots$ & $\ldots$ & $\ldots$ \\
\hline
\end{tabular}

Therefore, in terms of the four independent groups, the $4 \times 42$-D DCT can be further rearranged as:

$$
\begin{aligned}
G(m, n)=\frac{c(u) c(v)}{4} \sum_{0}^{3} \sum_{m}^{3} g_{m n}\left\{\cos \frac{(2 m+1) u \pi+(2 n+1) v \pi}{8}+\cos \frac{(2 m+1) u \pi-(2 n+1) v \pi}{8}\right\} \\
=\frac{c(u) c(v)}{4} \sum_{k=0}^{3}\left(X_{k}+Y_{k}\right)
\end{aligned}
$$

where:

$$
\begin{aligned}
& X_{k}=\sum_{m n} g_{m n} \cos \frac{(2 m+1) u \pi+(2 n+1) v \pi}{8} \\
& Y_{k}=\sum_{z_{n} \in x_{k}} g_{m n} \cos \frac{(2 m+1) u \pi-(2 n+1) \nu \pi}{8}
\end{aligned}
$$


and $x_{k}$ is the $k^{\prime}$ th group shown in the fifth column of Table I.

To find the best way of transforming the 2-D DCT into 1-D DCT, we represent the value of $n$ by $m,(3 m+1)(\bmod 4),(-3 m-2)(\bmod 4)$ and $(3 m+3)(\bmod 4)$ respectively. Corresponding to the four relationships, all the $4 \times 4$ input pixels are arranged into the following:

$$
\begin{array}{ll}
\mathrm{x}_{0}=\left\{\mathrm{g}_{00} \mathrm{~g}_{11} \mathrm{~g}_{22} \mathrm{~g}_{33}\right\} & \mathrm{x}_{1}=\left\{\mathrm{g}_{01} \mathrm{~g}_{10} \mathrm{~g}_{23} \mathrm{~g}_{32}\right\} \\
\mathrm{x}_{2}=\left\{\mathrm{g}_{02} \mathrm{~g}_{13} \mathrm{~g}_{20} \mathrm{~g}_{31}\right\} & \mathrm{x}_{3}=\left\{\mathrm{g}_{03} \mathrm{~g}_{12} \mathrm{~g}_{21} \mathrm{~g}_{30}\right\}
\end{array}
$$

By examining each group individually, the equations (4) and (5) can be rearranged into one dimensional DCTs for all the four groups as follows:

$$
\begin{aligned}
& X_{0}=D_{\left\{x_{0}\right)}(u+v) \quad Y_{0}=D_{\left(x_{0}\right)}(u-v) ;
\end{aligned}
$$

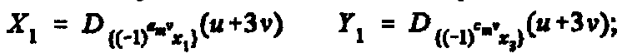

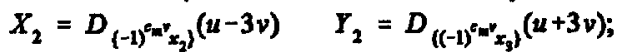

$$
\begin{aligned}
& X_{3}=D_{\left((-1)^{x_{3}}\right)}(u-v) \quad Y_{3}=D_{\left((-1)^{x_{3}}\right)}(u+v) \text {; }
\end{aligned}
$$

where:

$$
a_{m}=\left\{\begin{array}{ll}
0 & m=0 \\
1 & m=1 \\
1 & m=2 \\
2 & m=3
\end{array} \quad b_{m}= \begin{cases}1 & m=0 \\
2 & m=1 \\
2 & m=2 \\
3 & m=3\end{cases}\right.
$$

and $D_{(,)}(L)$ stands for the L'th 1-D DCT of the input elements \{\} .

As an example, since $n=3 m+3$ (modulo 4 ) is selected for group $x_{3}$, the modulo operation of $n$ can be eliminated by specifying:

$$
n=3 m+3-4 b_{m}
$$

The value of $b_{m}$ can be determined the same as $m$, in accordance with the modulo operation and all the pixels in group $\mathrm{x}_{3}$. Hence:

$$
2 n+1=6 m+6-8 m+1=-(2 m+1)+8
$$

Therefore $\mathrm{X}_{3}$ and $\mathrm{Y}_{3}$ can be obtained from equations (4) and (5) as follows:

$$
X_{3}=\sum_{m=0}^{3}(-1)^{n} g_{m n} \cos \left\{\frac{(2 m+1)(u-v) \pi}{8}\right\}=D_{\left((-1)^{4} x_{3}\right)}(u-v)
$$




$$
Y_{3}=\sum_{m \rightarrow 0}^{3}(-1)^{y} g_{m n} \cos \left\{\frac{(2 m+1)(u+v) \pi}{8}\right\}=D_{\left((-1)^{y} x_{3}\right)}(u+v)
$$

By putting the equations (6) into (3), the 2-D DCT can finally be arranged into the following form:

$$
\begin{aligned}
& G(m, n)=\frac{c(u) c(v)}{4} \sum_{k=0}^{3}\left(X_{k}+Y_{k}\right)=\frac{c(u) c(v)}{4}\left\{D_{\left(x_{0}+(-1)^{\prime} x_{2}\right)}(u+v)+D_{\left(x_{0}+(-1)^{v} x_{2}\right)}(u-v)\right.
\end{aligned}
$$

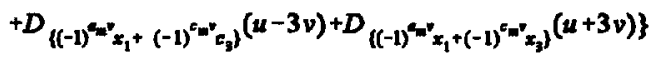

\begin{tabular}{|c|c|c|}
\hline$(u, v)$ & $G(u, v)(I)$ & $G(u, v)(I I)$ \\
\hline 0 & $\left\{\mathrm{D}_{1}(0)+\mathrm{D}_{1}(0)+\mathrm{D}_{3}(0)+\mathrm{D}_{3}(0)\right\}$ & $\left\{2 \mathrm{D}_{1}(0)+2 \mathrm{D}_{3}(0)\right\}$ \\
\hline 1 & $\left\{\mathrm{D}_{2}(1)+\mathrm{D}_{2}(1)+\mathrm{D}_{4}(3)+\mathrm{D}_{4}(3)\right\}$ & $\left\{2 \mathrm{D}_{2}(1)-2 \mathrm{D}_{4}(3)\right\}$ \\
\hline 2 & $\left\{\mathrm{D}_{1}(2)+\mathrm{D}_{1}(2)+\mathrm{D}_{3}(6)+\mathrm{D}_{3}(6)\right\}$ & $\left\{2 \mathrm{D}_{1}(2)+2 \mathrm{D}_{3}(6)\right\}$ \\
\hline 3 & $\left\{\mathrm{D}_{2}(3)+\mathrm{D}_{2}(3)+\mathrm{D}_{4}(9)+\mathrm{D}_{4}(9)\right\}$ & $\left\{2 \mathrm{D}_{2}(3)-2 \mathrm{D}_{4}(1)\right\}$ \\
\hline 10 & $\left\{\mathrm{D}_{1}(1)+\mathrm{D}_{1}(1)+\mathrm{D}_{3}(1)+\mathrm{D}_{3}(1)\right\}$ & $\left\{2 \mathrm{D}_{1}(1)+2 \mathrm{D}_{3}(1)\right\}$ \\
\hline 11 & $\left\{\mathrm{D}_{2}(2)+\mathrm{D}_{2}(0)+\mathrm{D}_{4}(1)+\mathrm{D}_{4}(1)\right\}$ & $\left\{\mathrm{D}_{2}(2)+\mathrm{D}_{2}(0)+2 \mathrm{D}_{4}(1)\right\}$ \\
\hline 12 & $\left\{\mathrm{D}_{1}(3)+\mathrm{D}_{1}(1)+\mathrm{D}_{3}(7)+\mathrm{D}_{3}(5)\right\}$ & $\left\{\mathrm{D}_{1}(3)+\mathrm{D}_{1}(1)-\mathrm{D}_{3}(1)-\mathrm{D}_{3}(3)\right\}$ \\
\hline 13 & $\left\{\mathrm{D}_{2}(4)+\mathrm{D}_{2}(2)+\mathrm{D}_{4}(10)+\mathrm{D}_{4}(8)\right\}$ & $\left\{\mathrm{D}_{2}(2)-\mathrm{D}_{4}(2)-\mathrm{D}_{4}(0)\right\}$ \\
\hline 20 & $\left\{\mathrm{D}_{1}(2)+\mathrm{D}_{1}(2)+\mathrm{D}_{3}(2)+\mathrm{D}_{3}(2)\right\}$ & $\left\{2 \mathrm{D}_{1}(2)+2 \mathrm{D}_{3}(2)\right\}$ \\
\hline 21 & $\left\{\mathrm{D}_{2}(3)+\mathrm{D}_{2}(1)+\mathrm{D}_{4}(5)+\mathrm{D}_{4}(1)\right\}$ & $\left\{\mathrm{D}_{2}(3)+\mathrm{D}_{2}(1)-\mathrm{D}_{4}(3)+\mathrm{D}_{4}(1)\right\}$ \\
\hline 22 & $\left\{\mathrm{D}_{1}(4)+\mathrm{D}_{1}(0)+\mathrm{D}_{3}(8)+\mathrm{D}_{3}(4)\right\}$ & $\left\{\mathrm{D}_{1}(0)-\mathrm{D}_{3}(0)\right\}$ \\
\hline 23 & $\left\{\mathrm{D}_{2}(5)+\mathrm{D}_{2}(1)+\mathrm{D}_{4}(11)+\mathrm{D}_{4}(7)\right\}$ & $\left\{-\mathrm{D}_{2}(3)+\mathrm{D}_{2}(1)-\mathrm{D}_{4}(3)-\mathrm{D}_{4}(1)\right\}$ \\
\hline 30 & $\left\{\mathrm{D}_{1}(3)+\mathrm{D}_{1}(3)+\mathrm{D}_{3}(3)+\mathrm{D}_{3}(3)\right\}$ & $\left\{2 \mathrm{D}_{1}(3)+2 \mathrm{D}_{3}(3)\right\}$ \\
\hline 31 & $\left\{\mathrm{D}_{2}(4)+\mathrm{D}_{2}(2)+\mathrm{D}_{4}(6)+\mathrm{D}_{4}(0)\right\}$ & $\left\{\mathrm{D}_{2}(2)-\mathrm{D}_{4}(2)+\mathrm{D}_{4}(0)\right\}$ \\
\hline 32 & $\left\{\mathrm{D}_{1}(5)+\mathrm{D}_{1}(1)+\mathrm{D}_{3}(9)+\mathrm{D}_{3}(3)\right\}$ & $\left\{-\mathrm{D}_{1}(3)+\mathrm{D}_{1}(1)-\mathrm{D}_{3}(1)+\mathrm{D}_{3}(3)\right\}$ \\
\hline 33 & $\left\{\mathrm{D}_{2}(6)+\mathrm{D}_{2}(0)+\mathrm{D}_{4}(12)+\mathrm{D}_{4}(6)\right\}$ & $\left\{-\mathrm{D}_{2}(2)+\mathrm{D}_{2}(0)-\mathrm{D}_{4}(2)\right\}$ \\
\hline
\end{tabular}

Table II Computation of $G(u, v)$ 


\section{A Complete Fast $4 \times 4$ DCT Algorithm}

When the value of $\mathrm{v}$ is considered as odd and even respectively, the two combinations in equation (9), $\left\{x_{0}+(-1)^{\prime} x_{2}\right\}$ and $\left\{(-1)^{a-1} x_{1}+(-1)^{c_{n}{ }^{*}} x_{3}\right\}$, can be further simplified into four combinations: $\left\{x_{0}+x_{2}\right\},\left\{x_{0}-x_{2}\right\},\left\{x_{1}+x_{3}\right\}$ and $\left\{(-1)^{3} x_{1}+(-1)^{c_{1}} x_{3}\right\}$.

Therefore, as the values of $u$ and $v$ vary from 0 to 3 , the complete $4 \times 4$ DCT can be computed, according to equation (9), as shown in Table II where $D_{1}(L), D_{2}(L), D_{3}(L)$ and $\mathrm{D}_{4}(\mathrm{~L})$ represent the 1-D DCT of the above four combinations respectively, such as $D_{1}(L)=D_{\left\langle x_{0}+F_{2}\right\}}(L)$ etc. From the final simplified results given in the second column in Table II, the complete flow chart for the algorithm to compute the 2-D DCT can be worked out as shown in Figure 1.

\section{Conclusions}

A fast and efficient 2-D $4 \times 4 \mathrm{DCT}$ algorithm has been developed and presented in this paper. The algorithm features one dimensional transformation and simple additions for the computation of 2-D DCT. This algorithm can also be applied to compute general 2-D $2^{\mathrm{m}} \times 2^{\mathrm{m}}$ DCT by recursive procedures. As the one dimensional DCT is the basic core for this algorithm, its efficiency in terms of computing complexity, such as the number of multiplications, additions, is

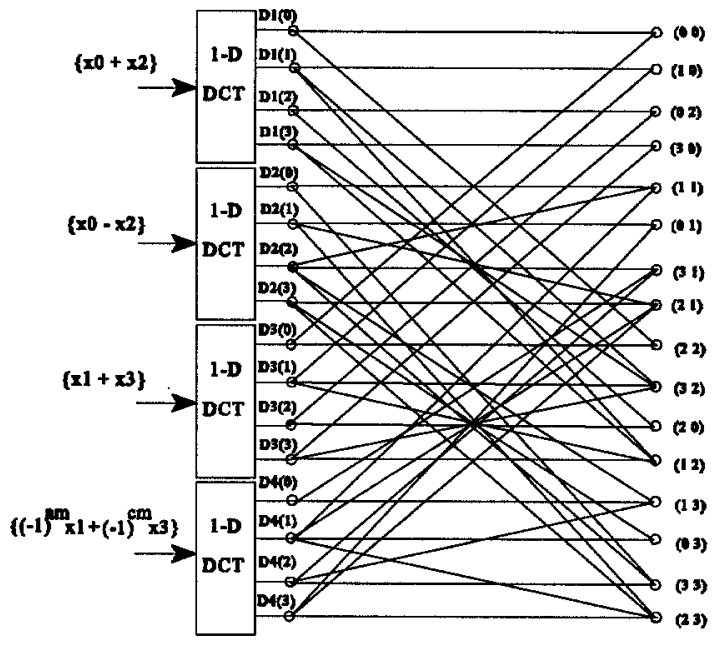

Figure 1 Flowchart of $4 \times 4$ DCT dependent on the 1-D DCT algorithm adopted. Therefore, all existing fast algorithm development for one dimensional DCT can be directly utilized to implement the 2-D DCT algorithm.

\section{References}

1. Rao, K.R. and Yip, P. Discrete cosine transform algorithms, advantages, and applications', Academic Press, INC. 1990, ISBN: 0-12-580203-x.

2. Hou, H.S. 'A fast recursive algorithm for computing the discrete cosine transform', IEEE Trans. Acoust., Speech, Signal Processing, Vol. ASSP-35, No. 10, 1455-1461, 1987.

3. Lee, B.G. 'A new algorithm to compute the discrete cosine transform' IEEE Trans. Acoust., Speech, Signal Processing, Vol. ASSP-32, No.6, 1243-1245, Dec. 1984.

4. Feig, Ephraim, Fast algorithms for the discrete cosine transform'. IEEE Trans. on Signal Processing, Vol. 40, No 9, 2174-2193, Sept. 1992. 